\title{
Impairment of EEG desynchronisation before and during movement and its relation to bradykinesia in Parkinson's disease
}

\author{
Han-Cheng Wang, Andrew J Lees, Peter Brown
}

\begin{abstract}
Objective-It has been suggested that the basal ganglia act to release cortical elements from idling ( $\alpha$ ) rhythms so that they may become coherent in the $\gamma$ range, thereby binding together those distributed activities necessary for the effective selection and execution of a motor act. This hypothesis was tested in 10 patients with idiopathic Parkinson's disease.

Methods-Surface EEG was recorded during self paced squeezing of the hand and elbow flexion performed separately, simultaneously, or sequentially. Recordings were made after overnight withdrawal of medication and, again, 1 hour after levodopa. The medication related improvement in EEG desynchronisation (in the 7.5-12.5 Hz band) over the 1 second before movement and during movement were separately correlated with the improvement in movement time for each electrode site. Correlation coefficients $(r)>0.632$ were considered significant $(\mathrm{p}<0.05)$.
\end{abstract}

Results-Improvement in premovement desynchronisation correlated with reduction in bradykinesia over the contralateral sensorimotor cortex and supplementary motor area in flexion and squeeze, respectively. However, when both movements were combined either simultaneously or sequentially, this correlation shifted anteriorly, to areas overlying prefrontal cortex. Improvement in EEG desynchronisation during movement only correlated with reduction in bradykinesia in two tasks. Correlation was seen over the supplementary motor area during flexion, and central prefrontal and ipsilateral premotor areas during simultaneous flex and squeeze.

Conclusions-The results are consistent with the idea that the basal ganglia liberate frontal cortex from idling rhythms, and that this effect is focused and specific in so far as it changes with the demands of the task. In particular, the effective selection and execution of more complex tasks is associated with changes over the prefrontal cortex.

(F Neurol Neurosurg Psychiatry 1999;66:442-446)

Keywords: Parkinson's disease; desynchronisation; bradykinesia

The cerebral cortical activity during rest and activity is characterised by oscillations in the $\alpha$ and $\gamma$ bands, respectively. The synchronisation of cortical elements in the $\gamma$ range is widely considered to be important in the binding together of particular aspects of distributed neuronal processing. ${ }^{1}$ Recently, it has been suggested that the basal ganglia (the caudate, lentiform and subthalamic nuclei, claustrum, substantia nigra, and dopaminergic neurons of the ventral tegmental area) act to release selected cortical elements from idling $\alpha$ rhythms so that they may become coherent (synchronised) in the $\gamma$ range. In this way, those channels of motor processing necessary to achieve a given movement may be favoured and bound together. ${ }^{1}$ The failure of this mechanism may contribute to the bradykinesia found in Parkinson's disease: the release of distributed cortical elements from idling activity is impaired so that those channels of motor processing necessary for an intended movement are no longer favoured and integrated through coherence in the $\gamma$ range. ${ }^{1}$

This hypothesis has recently been supported by the study of Brown and Marsden, in which the treatment related attenuation of those idling rhythms recorded over the cortical motor areas contralateral to the active arm correlated with the improvement in size and speed of movement effected by levodopa during a visual tracking task. ${ }^{2}$ However, this study had three drawbacks. It only examined changes during movement, recordings were not made from over the supplementary motor area, and the experimental task was limited to one that favoured processing in the lateral premotor system. ${ }^{34}$ Other studies have shown that EEG desynchronisation begins earlier before a self paced task in treated Parkinson's disease compared with untreated disease. ${ }^{5}{ }^{6}$ However, these studies failed to show a correlation between the increase in onset of desynchronisation or the degree of desynchronisation and the degree of clinical improvement. Here we investigate EEG changes over central and lateral motor areas during both the preparation and execution of self generated movements of varying complexity.

\section{Methods}

We studied 10 patients with Parkinson's disease (table 1). One, five, and four patients were Hoehn and Yahr stages I, II, and III, respectively, when off medication. All the patients were taking levodopa preparations (mean daily dosage $700 \mathrm{mg}$ ). Patients met criteria for the diagnosis of idiopathic Parkinson's disease $^{7}$ and had no clinical evidence of other neurological disease. All showed improvement 
Table 1 Clinical details of patients

\begin{tabular}{|c|c|c|c|c|c|c|c|c|}
\hline Patient & $\begin{array}{l}\text { Age and sex } \\
(y)\end{array}$ & $\begin{array}{l}\text { Duration of } \\
\text { Parkinsons's } \\
\text { disease }(y)\end{array}$ & $\begin{array}{l}\text { Hoehn and } \\
\text { Yahr stage* }\end{array}$ & $\begin{array}{l}\text { More } \\
\text { severe side }\end{array}$ & $\begin{array}{l}\text { UPDRS motor } \\
\text { score } \\
\text { Off/On }\end{array}$ & $\begin{array}{l}\text { Levodopa taken } \\
\text { during study } \\
\text { (mg) } \dagger\end{array}$ & $\begin{array}{l}\text { Total daily dose } \\
\text { levodopa of } \\
(\mathrm{mg})^{2}\end{array}$ & $\begin{array}{l}\text { Other } \\
\text { medications }\end{array}$ \\
\hline 1 & $57 \mathrm{M}$ & 1 & I & $\mathrm{L}$ & $6 / 1$ & 250 & 500 & Pergolide \\
\hline 2 & $70 \mathrm{M}$ & 4 & II & $\mathrm{R}$ & $10 / 5$ & 250 & 500 & None \\
\hline 3 & $74 \mathrm{M}$ & 5 & II & $\mathrm{L}$ & $8 / 2$ & 250 & 500 & None \\
\hline 4 & $58 \mathrm{M}$ & 12 & II & $\mathrm{R}$ & $13 / 9$ & 250 & 1125 & Tolcapone \\
\hline 5 & $71 \mathrm{~F}$ & 14 & III & $\mathrm{R}$ & $30 / 11$ & 250 & 750 & None \\
\hline 6 & $46 \mathrm{M}$ & 15 & III & $\mathrm{R}$ & $24 / 6$ & 250 & 750 & $\begin{array}{l}\text { Pergolide, } \\
\text { Selegiline }\end{array}$ \\
\hline 7 & $80 \mathrm{~F}$ & 3.5 & III & $\mathrm{R}$ & $15 / 9$ & 250 & 625 & None \\
\hline 8 & $53 \mathrm{~F}$ & 6.5 & II & $\mathrm{L}$ & $10 / 5$ & 200 & 700 & None \\
\hline 9 & $64 \mathrm{M}$ & 3 & III & $\mathrm{R}$ & $19 / 12$ & 125 & 375 & None \\
\hline 10 & $51 \mathrm{M}$ & 8.5 & II & $\mathrm{L}$ & $13 / 3$ & 375 & 1125 & None \\
\hline
\end{tabular}

$\star$ Off medication.

+With peripheral decarboxylase inhibitor.

of their UPDRS motor score on treatment with levodopa (mean treated and untreated scores 6 and 15 , respectively) and showed no significant EMG activity in scalp electrodes due to rest tremor or dyskinesia in the untreated and treated conditions. The worst affected hand was determined clinically and tested in each case (left hand in four patients). Records were made after overnight withdrawal of antiparkinsonian medication, and again 1 hour after treatment was restarted on the same day. All studies were performed with the approval of the local ethics committee and the informed consent of each subject.

Scalp EEG was recorded with $9 \mathrm{~mm}$ diameter silver-silver chloride electrodes, fixed with collodion. F3, FC3, C3, P3, Fz, FCz, Cz, $\mathrm{F} 4, \mathrm{FC} 4, \mathrm{C} 4$, and $\mathrm{P} 4$ were referenced to linked ears. Of these, F3/4 and Fz overlie the dorsal prefrontal cortex, and $\mathrm{FCz}$ and $\mathrm{Cz}$ overlie the supplementary motor area, whereas $\mathrm{FC} 3 / 4$, $\mathrm{C} 3 / 4$, and $\mathrm{P} 3 / 4$ overlie the premotor areas, sensorimotor cortices, and the superior parietal lobules, respectively. ${ }^{89}$

Subjects were seated with the forearm of the tested hand resting on a manipulandum. The experimental set up has been previously described in detail. ${ }^{10-12}$ The angular position of the elbow was monitored by a potentiometer. A $\mathrm{U}$ shaped bar was grasped between the thumb and fingers. A strain gauge was mounted on the U shaped bar so that the force of squeeze could be monitored. EEG signals were amplified and band pass filtered between $0.5 \mathrm{~Hz}$ and $300 \mathrm{~Hz}$. Force and positional signals were amplified and band pass filtered between DC and $300 \mathrm{~Hz}$. They were digitised with 12 bit resolution, and collected and analysed on a PC by a software package (CED spike 2). The sampling rate was $640 \mathrm{~Hz}$.

Subjects were asked to perform four different tasks with their worst affected upper limb: (1) flex the elbow joint as rapidly as possible through an angle of 30 degrees from a starting angle of 135 degrees ("flex"); (2) squeeze the strain gauge as rapidly as possible to a force of 20 N ("squeeze"); (3) execute both tasks simultaneously as rapidly as possible ("flex and squeeze"); (4) "squeeze" then "flex", as rapidly as possible, and start the "flex" immediately after the end of the "squeeze" ("squeeze then flex"). Elbow position and grip force were displayed on an oscilloscope before the subjects as visual feedback. Each movement was practised about five times before data collection. Ten single trials of each type of movement were collected, with 2 minutes of rest between each run. Within a run, movements were self paced, at intervals of about $10-16$ seconds.

Data were inspected off line on a computer screen. EEG data with artefact due to EMG or eye movement were rejected. Cursors were used to measure the total duration of the flex, squeeze, flex and squeeze, and squeeze then flex movements and to select periods for spectral analysis. Movement times were measured from the onset of the movement to the crossing of the baseline of the same movement in the flex and squeeze tasks and to the crossing of the baseline of the second or longest duration movement in the squeeze then flex and squeeze and flex tasks (see vertical lines in fig 1). Movement times measured on and off treatment were compared by the paired $t$ test. The fast Fourier transform was used to compute the discrete Fourier transform of blocks of EEG data. Blocks were of equal duration (256 data points), and spectra were estimated by averaging across blocks. The non-cycling nature of data blocks was dealt with by applying a raised cosine window to each block, and then compensating for the resultant loss of power.

EEG amplitude spectra were calculated for three different periods in each run: rest (defined as the period at least 3 seconds after the last movement and not less than 3 seconds before the next movement), premovement (defined as the 1 second period before the onset of each movement) and movement (the same period as measured for the movement time). These were averaged for each movement type in each subject. The percentage change in the $\alpha(7.5 \mathrm{~Hz}-12.5 \mathrm{~Hz})$ band in the premovement period was calculated according to the formula:

\section{(rest-premovement/rest) $\times 100$}

Similar calculations were performed for the movement periods. Correlation coefficients $(r)$ for $\%$ change in $\alpha$ band treated- $\%$ change in $\alpha$ band untreated versus (untreated movement time- treated movement time/untreated movement time $) \times 100$ ) were calculated for premovement and movement periods for each electrode site. A p value $<0.05$ (one tailed) was taken as significant, whenever correlation was positive (constrained to our a priori hypothesis that levodopa related improvement in EEG 
Table 2 Mean (SD) of movement times on and off medication in the 10 patients

\begin{tabular}{cllll}
\hline & Squeeze & Flex & Squeeze and flex & Squeeze then flex \\
\hline Off:: & & & & \\
Mean & 0.8013 & 1.6695 & 2.288 & 2.9113 \\
SD & 0.33339 & 0.40801 & 0.87601 & 1.17246 \\
On: & & & & \\
Mean & 0.6339 & 1.3369 & 1.6932 & 2.1644 \\
SD & 0.31982 & 0.35611 & 0.66136 & 0.82008 \\
p Value & 0.00119 & 0.00224 & 0.00386 & 0.00551 \\
\hline
\end{tabular}

The movement times on and off are compared by paired $t$ test for each task.

desynchronisation would correlate with improvement in bradykinesia).

\section{Results}

Figure 1 gives examples of the attenuation of $\alpha$ activity 1 second before and during the four tasks in a parkinsonian patient after withdrawal (off) and reintroduction of levodopa (on). Treatment with levodopa enhanced the attenuation of the idling rhythms, and reversed the bradykinesia evident when the task was performed off medication. Levodopa improved movement time by $20 \%$ to $26 \%$ (table 2 ).

For the flex task across all 10 cases, the treatment related premovement EEG desynchronisation and improvement in movement time was correlated over the C3/4 electrode contralateral to the active limb $(r=0.660$, $\mathrm{p}<0.05)$. For the squeeze task, the main correlation was over $\mathrm{FCz} \quad(r=0.647, \mathrm{p}<0.05)$, although there was still a also tendency to correlation over the contralateral sensorimotor cortex $(r=0.501, \mathrm{NS})$. For the simultaneous flex and squeeze task, the correlation was over $\mathrm{Fz} \quad(r=0.636, \mathrm{p}<0.05)$. For the sequential squeeze then flex task, correlation was seen over F3, Fz, and F4 $(r=0.744,0.661$, and $0.688, \mathrm{p}<0.05)$. These findings are summarised in fig $2 \mathrm{~A}-\mathrm{D}$.

For the flex task across all 10 cases, the treatment related movement EEG desynchronisation and improvement in movement time was correlated over $\mathrm{FCz} \quad(r=0.757, \mathrm{p}<0.05)$ and $\mathrm{Cz}(r=0.716, \mathrm{p}<0.05)$. For the flex and squeeze task, correlation was present over $\mathrm{Fz}$ $(r=0.641, \quad \mathrm{p}<0.05)$ and ipsilateral FC3/4 $(r=0.654, \mathrm{p}<0.05)$. However, no significant correlation was found for the squeeze and the squeeze then flex tasks. These findings are summarised in fig $2 \mathrm{E}-\mathrm{H}$.

\section{Discussion}

Patients with Parkinson's disease may have abnormal patterns of movement related attenuation of the $\alpha$ and $\beta$ rhythms, which can be partially reversed by dopaminergic stimulation..$^{25611}$ This study is the first to show a correlation between the treatment related attenuation of the $\alpha$ rhythm over cortical motor areas contralateral to the active arm before movement and the improvement in bradykinesia effected by levodopa. Correlation was also shown between EEG desynchronisation during movement and improvement in bradykinesia. Correlations were focal and varied with each task. They were not, therefore, simply due to a direct pharmacological effect of levodopa or its metabolites on the cerebral cortex. Nor can the changes with levodopa be considered a general feature of brain activation around the time of movement: correlations were conspicuously absent over the sensorimotor cortex in all but one task.

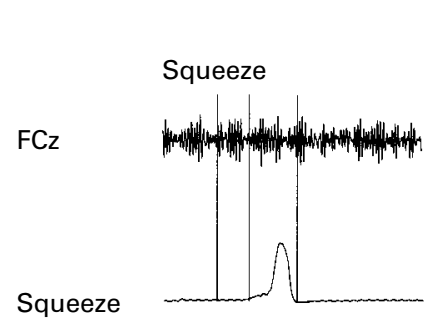

Flex
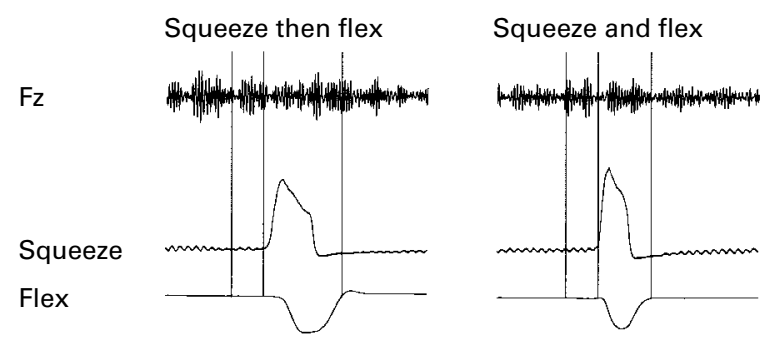

ON
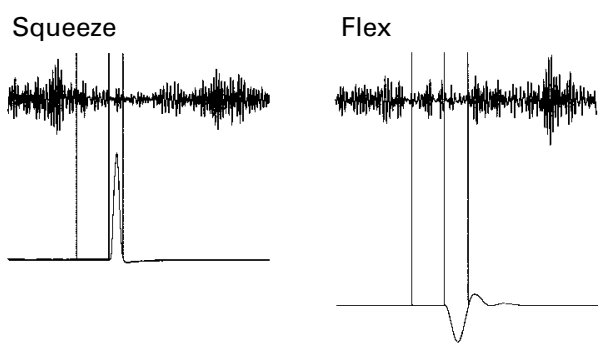

Squeeze then flex

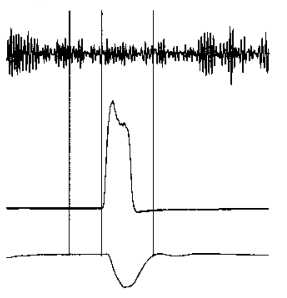

Figure 1 Examples (single trials) of the attenuation of a activity 1 second before and during the tasks in a parkinsonian patient after drug withdrawal (off) and reintroduction of levodopa (on). The EEG has been digitally band pass filtered from 7.5-12.5 Hz. FCz and Fz have been selected, as these channels showed the greatest change with levodopa across subjects in the single and combined tasks, respectively (see fig 2). Force and position traces are shown for hand squeeze and elbow flexion, respectively. Thin vertical lines have been drawn to indicate the onset of movement (second line), the premovement period (first to the second line) and the period measured as the movement time (second to the third line). Treatment with levodopa in this patient enhanced the attenuation of the idling rhythms around the time of movement, and reversed the bradykinesia evident when the task was performed without medication. Note the rest tremor in the squeeze trace in the off state. 
A Flex

PREMOVEMENT

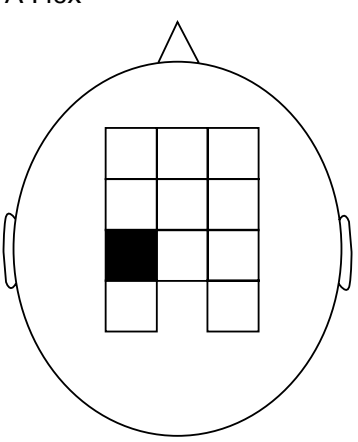

B Squeeze

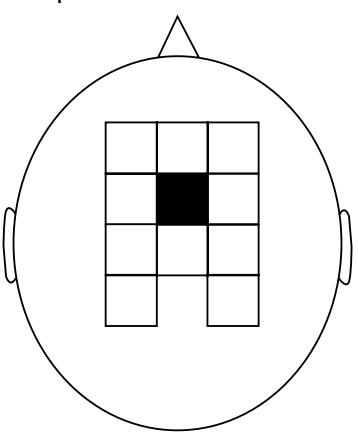

C Flex and squeeze

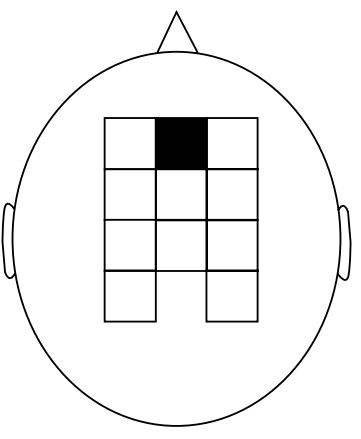

D Squeeze then flex

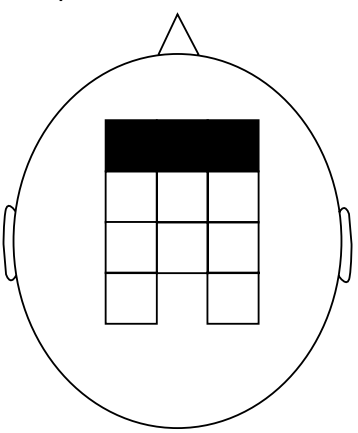

MOVEMENT

E Flex

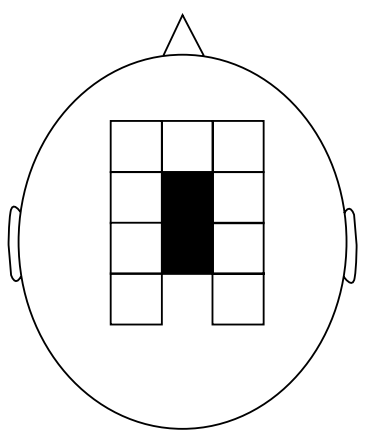

F Squeeze

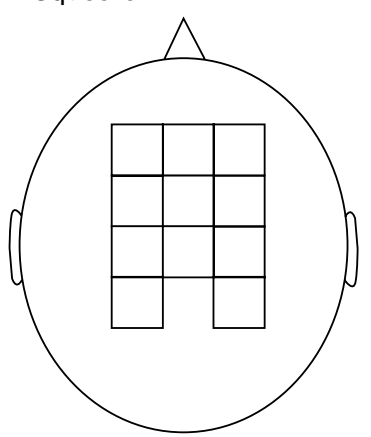

G Flex and squeeze

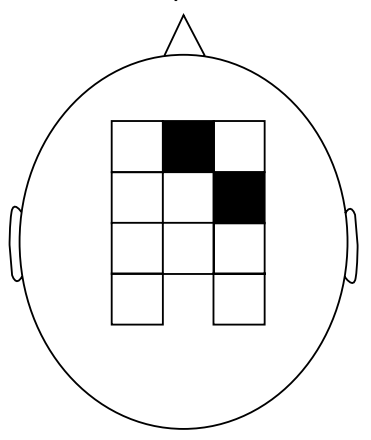

H Squeeze then flex

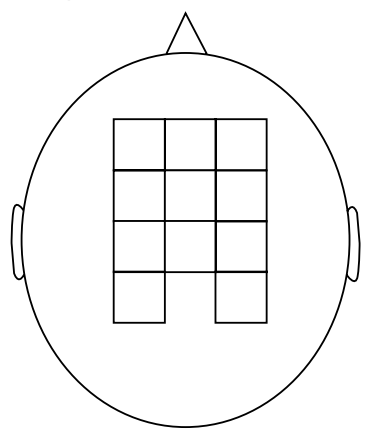

Figure 2 Correlation between levodopa induced EEG desynchronisation and improvement in movement time. The data have been normalised to right hand movement (EEG records from left and right sides of the head were reversed before analysis in the four patients in whom the left hand was tested). Each small square represents an electrode site, and the left and right sided squares represent electrode sites contralateral and ipsilateral to the tested limb. The black squares denote electrode sites where there is a significant correlation $(r>0.632, n=10, p<0.05)$ between levodopa induced EEG desynchronisation and improvement in movement time. A to $D$ : correlation over the 1 second before movement (premovement) in each of the four tasks. $E$ to $H$ : correlation during movement in each of the four tasks.

Our findings support the theory that the basal ganglia help to free from idling rhythms those cortical elements necessary for the selection, preparation, and execution of a given motor act. ${ }^{1}$ These elements may then become coherent in the $\gamma$ band, and are thus favoured and bound together, so that a complete action may be efficiently achieved. Untreated Parkinson's disease may lead to the impaired release of cortical elements from idling activities. This compromises subsequent processing so that movement occurs incompletely and slowly. Indirect evidence for the impairment of cortical $\gamma$ activity-the corollary of the basal ganglia driven EEG desynchronisationcomes from recordings of muscle. Normally, voluntary movement is accompanied by $\gamma$ band activity in the motor cortex that is coherent with parallel rhythmic activity in muscle. ${ }^{14}$ These muscle oscillations at around $40 \mathrm{~Hz}$ are lost in bradykinetic parkinsonian patients withdrawn from levodopa, and restored by reintroduction of that drug. ${ }^{15}$

The correlations between the levodopa related EEG dysynchronisation and improvement in bradykinesia seen in the present study were mainly over the supplementary motor area and dorsal prefrontal cortex. Current theories of motor control associate the activation of dorsal prefrontal and supplementary motor areas with internally cued actions and motor sequences, whereas activation of the lateral premotor area is more associated with externally triggered movements. ${ }^{3}{ }^{416-20}$ A comparison of this study with that of Brown and Marsden $^{2}$ seems to bear out this broad division. Thus in the tracking study of Brown and Marsden there was much more correlation over the premotor area and superior parietal lobule, whereas in the present study, in which movements were self generated, supplementary motor and prefrontal changes seemed to dominate. No changes were seen over the superior parietal lobule and very little change was seen over the premotor area in the present experiments. This distinction is important as it suggests that the effect of the basal ganglia on idling rhythms changes according to task in a functionally relevant way. In this regard, the differing cortical distributions of the correlations in the flex and squeeze tasks are also interesting. Simple elbow flexion was associated with a correlation between reduction in bradykinesia and premovement desynchronisation over the contralateral sensorimotor cortex, whereas squeeze, which involved the coordinated action of many intrinsic hand and forearm muscles, was associated with correlation over the supplementary motor area.

Practical considerations limited the usefulness of analyses of desynchronisation during the movement tasks used here. Squeeze was brief and the absence of correlation between EEG and movement changes might reflect an inadequate sample. In addition, there was no correlation between EEG and movement changes in the squeeze then flex task. This may be due to variability in the interonset latency both within and between subjects and due to the fact that both premovement and movement related desynchronisation, and postmovement related synchronisation ${ }^{21-23}$ would have been present during the period of measurement. 
The improvements in EEG desynchronisation for complex movements were not a simple addition of their parts, as has been noted in comparable Bereitschaftspotential studies. ${ }^{24}$ This was most clearly seen in the premovement period of the squeeze then flex task in which dorsal prefrontal cortex was activated bilaterally. In separate flex and squeeze tasks the contralateral hand area and supplementary motor area were activated. A similar distinction was seen in the movement phase for the simultaneous flex and squeeze task, as opposed to flex and squeeze alone. Again, correlations shifted forwards to prefrontal cortex in the combined movement. It has previously been suggested that flex and squeeze tasks are organised as separate motor programmes during combined movements. This is based on the finding that there is no correlation between the movement times of flex and squeeze in the simultaneous and sequential tasks. ${ }^{1012}$ In this case, the prefrontal changes seen in the more complex tasks must be related to the extra processing entailed in the superimposition and sequencing of motor programmes over and above what is necessary to run each single programme. Benecke $e t a l^{10-12}$ have defined these additional activities as motor planning. The present results are important in suggesting that the motor planning activity related to complex movements is centred on the dorsal prefrontal cortex and is dependent, at least in part, on input from the basal ganglia. Specifically, normal functioning of the basal ganglia may release relevant elements in the dorsal prefrontal cortex from the idling state. This influence could be exerted by direct mesocortical dopaminergic projections to the prefrontal $\operatorname{cortex}^{25}$ or via the prefrontal basal gangliathalamocortical circuit. ${ }^{26}$

Our results confirm a selective and focal effect of levodopa on the organisation of motor cortical activity in the frequency domain during the planning and execution of movement-an effect that correlates with improvements in bradykinesia. The results are consistent with the idea that the basal ganglia liberate the motor areas of the frontal cortex from idling rhythms, and that this effect is focused and specific in so far as it changes with the demands of the task. In particular, the effective selection, preparation, and execution of more complex tasks are associated with changes over the prefrontal cortex.

We thank Mr R Bedlington and Mr P Asselman for their technical assistance.

1 Brown P, Marsden CD. What do the basal ganglia do? Lancet 1998;351:801-4.
2 Brown P, Marsden CD. EEG desynchronisation during Brown P, Marsden CD. EEG desynchronisation during Disord 1998;13:35.

3 Passingham RE. The frontal lobes and voluntary action. Oxford: Oxford University Press 1993

4 Goldberg G. Supplementary motor area structure and function: review and hypotheses. Behavioral and Brain Sciences 1985;8:567-615.

5 Magnani G, Cursi M, Leocani L, et al. Event-related desynchronisation in Parkinson's disease: effect of acute administration of L-DOPA. Electroencephalogr Clin Neurophysiol 1997;103:156.

6 Defebvre L, Bourriez JL, Derambure $\mathrm{Ph}$, et al. Influence of chronic administration of L-DOPA on event-related desynchronisation of mu rhythm preceding voluntary movement in Parkinson's disease. Electroencephalogr Clin Neurophysiol in Parkinson's dise

7 Hughes AJ, Daniel SE, Kilford L, et al. Accuracy of clinical diagnosis of idiopathic Parkinson's disease: a clinicopathological study of 100 cases. F Neurol Neurosurg Psychiatry 1992;55:181-4.

8 Steinmetz H, Fürst G, Meyer BU. Craniocerebral topography within the international 10-20 system. Electroencephalogr Clin Neurophysiol 1989;72:499-506.

9 Homan RW, Herman J, Purdy P. Cerebral location of international 10-20 system electrode placement. Electroencephalogr Clin Neurophysiol 1987;66:376-82.

10 Benecke R, Rothwell JC, Dick JP, et al. Performance of simultaneous movements in patients with Parkinson's disease. Brain 1986;109:739-57.

11 Benecke R, Rothwell JC, Dick JP, et al. Simple and complex movements off and on treatment in patients with Parkinson's disease. F Neurol Neurosurg Psychiatry 1987;50: 296-303.

12 Benecke R, Rothwell JC, Dick JPR, et al. Disturbances of sequential movements in patients with Parkinson's disease. Brain 1987;110:361-79.

13 Defebvre L, Derambure P, Bourriez JL, et al. Spatiotemporal study of event-related desynchronisation in idiopathic Parkinson's disease. Adv Neurol 1993;60:422-8.

14 Brown P, Salenius S, Rothwell JC, et al. The origin of the Piper rhythm in man. $\mathcal{f}$ Physiol 1998;507:34P.

15 Brown P. Muscle sounds in Parkinson's disease. Lancet 1997;349:533-35

6 Halsband U, Matsuzaka Y, Tanji J. Neuronal activity in the primate supplementary, pre-supplementary and premotor cortex during externally and internally instructed sequential movements. Neurosci Res 1994;20:149-55.

17 Mushiake H, Inase M, Tanji J. Neuronal activity in the primate premotor, supplementary, and precentral motor cortex during visually guided and internally determined sequential movements. I Neurophysiol 1991;66:705-18.

18 Okano K, Tanji J. Neuronal activities in the primate motor fields of the agranular frontal cortex preceding visually triggered and self-paced movement. Exp Brain Res triggered and self-

19 Winstein CJ, Grafton ST, Pohl PS. Motor task difficulty and brain activity: investigation of goal-directed reciprocal aiming using positron emission tomography. $\mathcal{F}$ Neurophysiol 1997;77:1581-94.

20 Jahanshahi M, Jenkins IH, Brown RG, et al. Self-initiated versus externally triggered movements. I. An investigation using measurement of regional cerebral blood flow with PET and movement-related potentials in normal and Parkinson's disease subjects. Brain 1995;118:913-33.

21 Leocani L, Toro C, Manganotti P, et al. Event-related coherence and event-related desynchronization/ synchronization in the $10 \mathrm{~Hz}$ and $20 \mathrm{~Hz}$ EEG during selfpaced movements. Electroencephalogr Clin Neurophysiol paced movements.

22 Pfurtscheller G, Stancak A, Neuper C. Post-movement $\beta$ synchronisation. A correlate of an idling motor area? synchronisation. A correlate of an idling motor
Electroencephalogr Clin Neurophysiol 1996;98:281-93.

23 Salmelin R, Forss N, Knuutila J, et al. Bilateral activation of the human somatomotor cortex by distal hand movements. Electroencephalogr Clin Neurophysiol 1995;95:444-52.

24 Benecke R, Dick JPR, Rothwell JC, et al. Increase in the Bereitschaftspotential in simultaneous and sequential movements. Neurosci Lett 1985;62:347-52.

25 Agid Y, Javoy-Agid F, Ruberg M. Biochemistry of neurotransmitters in Parkinson's disease. In: Marsden CD, Fahn S, eds. Movement disorders 2. London: Butterworths, 1987:166-230.

26 Alexander GE, Crutcher MD, Delong MR. Basal ganglia thalamo-cortical circuits: parallel substrates for motor, oculomotor, prefrontal and limbic functions. Prog Brain Res 1990;85:119-46. 